\title{
Probe-diverse Ptychography
}

\author{
I. Peterson ${ }^{\mathrm{a}}$, R. Harder ${ }^{\mathrm{b}}$, I.K. Robinson ${ }^{\mathrm{c}}$
}

${ }^{a}$ ARC Centre of Excellence for Coherent X-ray Science, the University of Melbourne, School of Physics, Victoria, 3010, Australia

${ }^{b}$ Advanced Photon Source, Argonne National Laboratory, Argonne, IL 60439, USA

${ }^{c}$ Research Complex at Harwell, Didcot, Oxfordshire OX11 ODE, UK London Centre for Nanotechnology, University College London, London, WC1H OAH, UK

\begin{abstract}
We propose an extension of ptychography where the target sample is scanned separately through several probes with distinct amplitude and phase profiles and a diffraction image is recorded for each probe and each sample translation. The resulting probediverse dataset is used to iteratively retrieve high-resolution images of the sample and all probes simultaneously. The method is shown to yield significant improvement in the reconstructed sample image compared to the image obtained using the standard single-probe ptychographic phase-retrieval scheme.
\end{abstract}

\section{Introduction}

Ptychography [1, 2, 3], is a recent extension of Coherent Diffractive Imaging (CDI) that promises to deliver atom-scale imaging of extended samples without the restrictions imposed by an objective lens. In the standard ptychographic scheme a ptychographic diffraction dataset is obtained by translating the sample across a localised, coherent probe, in steps small enough to ensure that the illuminated sample regions are sufficiently overlapped [4]. It has also been shown that the image can be reconstructed using data obtained by scanning the sample longitudinally through the probe $[5,6]$. Overlapping the illuminated regions of the sample provides a high level of redundancy in the ptychographic dataset and can significantly improve the accuracy and robustness in the sample image retrieval compared to standard CDI, with the ability to recover high-resolution, quantitative images of the probe and sample over an extended field of view. As a consequence, there has been significant interest in using ptychography with an X-ray probe $[1,3]$, in conjunction with tomography in three dimensional imaging [7, 8], using an electron probe $[9,10,11]$, and in other applications including optical encryption $[12,13]$.

Although overlapping the illuminated regions of the sample can assist in the sample image retrieval, the reconstruction algorithms can stagnate due to a number of issues including deviations from perfect spatial coherence [1], errors in the recorded ptychographic scan trajectory [14], and issues relating to sampling $[15,16]$. These issues can result in image artefacts or the complete failure in algorithm convergence. A number of algorithms have been suggested to address these, and other problems $[14,15,16,17,18,19]$. It has also been suggested that additional diversity can be introduced through adjusting the probe $[5,20]$ can yield improved results compared to the those obtained using the standard single-probe configuration.

In this paper we present an experimental demonstration of a method that can use a mixed ptychographic diffraction dataset that is obtained by scanning the sample separately through sev- eral probes with distinct amplitude and phase profiles. The resulting "probe-diverse" ptychographic dataset is obtained by recording a diffraction image for each probe and each sample translation. The dataset is used in conjunction with a modified version of the ePIE algorithm to retrieve quantitative images of the sample and all illuminating probes simultaneously. The algorithm is tested with Synchrotron X-ray diffraction data, yielding a significant improvement in the probe and sample reconstructions compared to those achieved with any of the standard single-probe reconstructions.

\section{Methods}

The proposed probe-diverse ptychographic algorithm can use ptychographic diffraction data generated from $N$ distinct probes, $\left\{P_{1}(\mathbf{r}), \ldots, P_{N}(\mathbf{r})\right\}$, incident on a target sample with sample transmission function, $O(\mathbf{r})$, where $\mathbf{r}$ is a co-ordinate vector in the sample plane. The sample is scanned through each probe separately over $N$ scanning trajectories, $\left\{\mathbf{s}_{1}, \ldots, \mathbf{s}_{N}\right\}$, and a diffraction image is recorded for each sample translation. In reference to the standard single-probe ptychographic scheme this yields $N$ distinct ptychographic datasets. Here all of the ptychographic data is combined into a single probe-diverse dataset that is used to reconstruct the sample and all probes simultaneously.

With the sample at the $j^{\text {th }}$ scan position of the $m^{\text {th }}$ scanning trajectory, $\mathbf{s}_{m, j}$, the exit surface wave, $\psi_{m, j}(\mathbf{r})$, is given by

$$
\psi_{m, j}(\mathbf{r})=O\left(\mathbf{r}-\mathbf{s}_{m, j}\right) P_{m}(\mathbf{r}) .
$$

The far-field wavefield, $\Psi_{m, j}(\mathbf{k})$, where $\mathbf{k}$ is a far-field coordinate vector, is obtained via the two dimensional Fourier transform, of the exit surface wave, i.e. $\Psi_{m, j}(\mathbf{k}) \propto \mathbb{F}\left[\psi_{m, j}(\mathbf{r})\right]$. An updated estimate of $\Psi_{m, j}(\mathbf{k})$ is obtained by enforcing consistency with the recorded diffraction data, $I_{m, j}(\mathbf{k})$, via the modulus constraint, defined as

$$
\Psi_{m, j}^{\prime}(\mathbf{k})=\Psi_{m, j}(\mathbf{k}) \frac{\sqrt{I_{m, j}(\mathbf{k})}}{\left|\Psi_{m, j}(\mathbf{k})\right|} .
$$


The accuracy of the current wavefield estimate can be measured using the $\chi^{2}$ metric, where

$$
\chi_{m, j}^{2}=\frac{\sum_{\mathbf{k}}\left[\left|\Psi_{m, j}(\mathbf{k})\right|-\sqrt{I_{m, j}(\mathbf{k})}\right]^{2}}{\sum_{\mathbf{k}} I_{m, j}(\mathbf{k})} .
$$

The updated wavefield, $\Psi_{m, j}^{\prime}(\mathbf{k})$, is then propagated back to the sample plane where the sample transmission function, $O(\mathbf{r})$, and the $m^{\text {th }}$ probe, $P_{m}(\mathbf{r})$, are updated according to the overlap constraint suggested by Maiden et al. [2]. The current estimate of the scattered wavefield is then propagated back to the sample plane and where the algorithm enforces consistency with the diffraction data obtained by the subsequent probe, $P_{m+1}(\mathbf{r})$. The iterative application of the above steps enables the recovery of the sample image and all illuminating probes simultaneously.

\section{Experiment}

The algorithm was tested using X-ray data obtained at the Advanced Photon Source (APS) beamline 34-ID-C using an experimental geometry detailed elsewhere [21]. All measurements were conducted using a $9 \mathrm{keV}$ beam $(\lambda=0.138 \mathrm{~nm})$.

The distinct probes were obtained by adjusting the slit width of a set of probe-defining slits positioned upstream of the sample and approximately $48 \mathrm{~m}$ downstream from the undulator. The probe-defining slits were initially set to widths of $10 \mu \mathrm{m} \times$ $20 \mu \mathrm{m}$ in the horizontal and vertical directions respectively. The resulting probe was focussed onto the target sample using a set of Kirkpatrick-Baez (KB) mirrors. The horizontal and vertical focussing mirrors were positioned $0.12 \mathrm{~m}$ and $0.22 \mathrm{~m}$ upstream of the sample stage respectively, with the angle of incidence set to approximately 3 milliradians relative to the beam. The sample, shown in the SEM image in Fig. 1, was manufactured by depositing a $1.5 \mu \mathrm{m}$ thick tungsten layer onto a silicon nitride support membrane. A series of star shaped apertures were lithographed into the tungsten layer. Errors in the manufacture resulted in small $(\sim 100 \mathrm{~nm})$ tungsten remnants on the star spokes. These are seen as a series of "spots" in the magnification in Fig. 1 (b). At $9 \mathrm{keV}$ the sample attenuated $70 \%$ of the probe [22], with full transmission through the lithographed regions.

The star shaped aperture was scanned through the probe using an $x y z$ nPoint NPXY100Z25A piezo scanning stage. The scan trajectory covered a $10 \mu \mathrm{m} \times 10 \mu \mathrm{m}$ area, consisting of 323 scanning points arranged on a series of concentric circles, with a $0.5 \mu \mathrm{m}$ radial increment between adjacent rings. This scanning pattern has been shown to reduce scanning trajectory artefacts associated with a raster scanning grid [23]. The diffraction dataset was recorded $3.2 \mathrm{~m}$ downstream from the test sample using a Timepix photon-counting detector with $55 \mu \mathrm{m}$ square pixels. 30 images were recorded and summed for each ptychographic scan position. The data acquisition time for each image was 0.04 seconds. Additional ptychographic datasets were recorded using slit widths of $20 \mu \mathrm{m} \times 40 \mu \mathrm{m}$ and $40 \mu \mathrm{m} \times$ $50 \mu \mathrm{m}$. Aside from the increase in slit width, the remaining experimental parameters for both datasets were those used in


Figure 1: (a) A SEM image of the star shaped aperture lithographed into a $1.5 \mu \mathrm{m}$ tungsten layer. Errors in the lithography resulted in tungsten remnants on the star spokes, seen as a series of small $(\sim 100 \mathrm{~nm})$ "spots" in the magnification in (b).

the $10 \mu \mathrm{m} \times 20 \mu \mathrm{m}$ dataset acquisition, in particular the sample was scanned through the same trajectory for each acquired dataset. The region of interest in all diffraction data was set to a $256 \times 256$ array centred on the diffraction peak. The experimental parameters resulted in a sample plane sampling interval of $31 \mathrm{~nm}$.

\section{Results}

The sample transmission function and probe were retrieved using the $10 \mu \mathrm{m} \times 20 \mu \mathrm{m}$ dataset and the standard ePIE algorithm with a sampling correction [16] for a total of 500 iterations. The initial estimate for the sample transmission function was a random binary array, with an initial probe estimate based on a knife edge scan of the central lobe. The resulting reconstructed sample transmission phase and amplitude are shown in Fig. 2 (a) and (b) respectively. The retrieved sample transmission phase is presented in phase-wrapped form as the rapid (and erroneous) phase transitions introduced additional errors when using phase-unwrapping algorithms. The reconstructed probe amplitude is shown in Fig. 2 (e). The normalized net flux density of the probe, obtained by superimposing the normalized probe amplitude at each point in the scan trajectory, is shown in Fig. 2 (f).

The sample transmission phase and amplitude both exhibit a strongly correlated erroneous variation in the lithographed regions, known to be of uniform density. An additional 500 iterations yielded no qualitative improvement in the sample image and no reduction in the $\chi^{2}$ metric (Eq. 2). The reconstructions were tested using 10 distinct random binary initial estimates of the sample transmission function, yielding artefacts in the sample transmission image that were inconsistent between distinctly initialised reconstructions. This is an indication of a local (but not global) minimisation of the $\chi^{2}$ metric for each reconstruction. The overlap between adjacent probe positions was $68.8 \%$.

The sample transmission function and probe were also reconstructed using the ePIE algorithm and the $20 \mu \mathrm{m} \times 40 \mu \mathrm{m}$ dataset. The resulting (phase-wrapped) sample transmission phase and amplitude reconstructions are provided in Fig. 2 

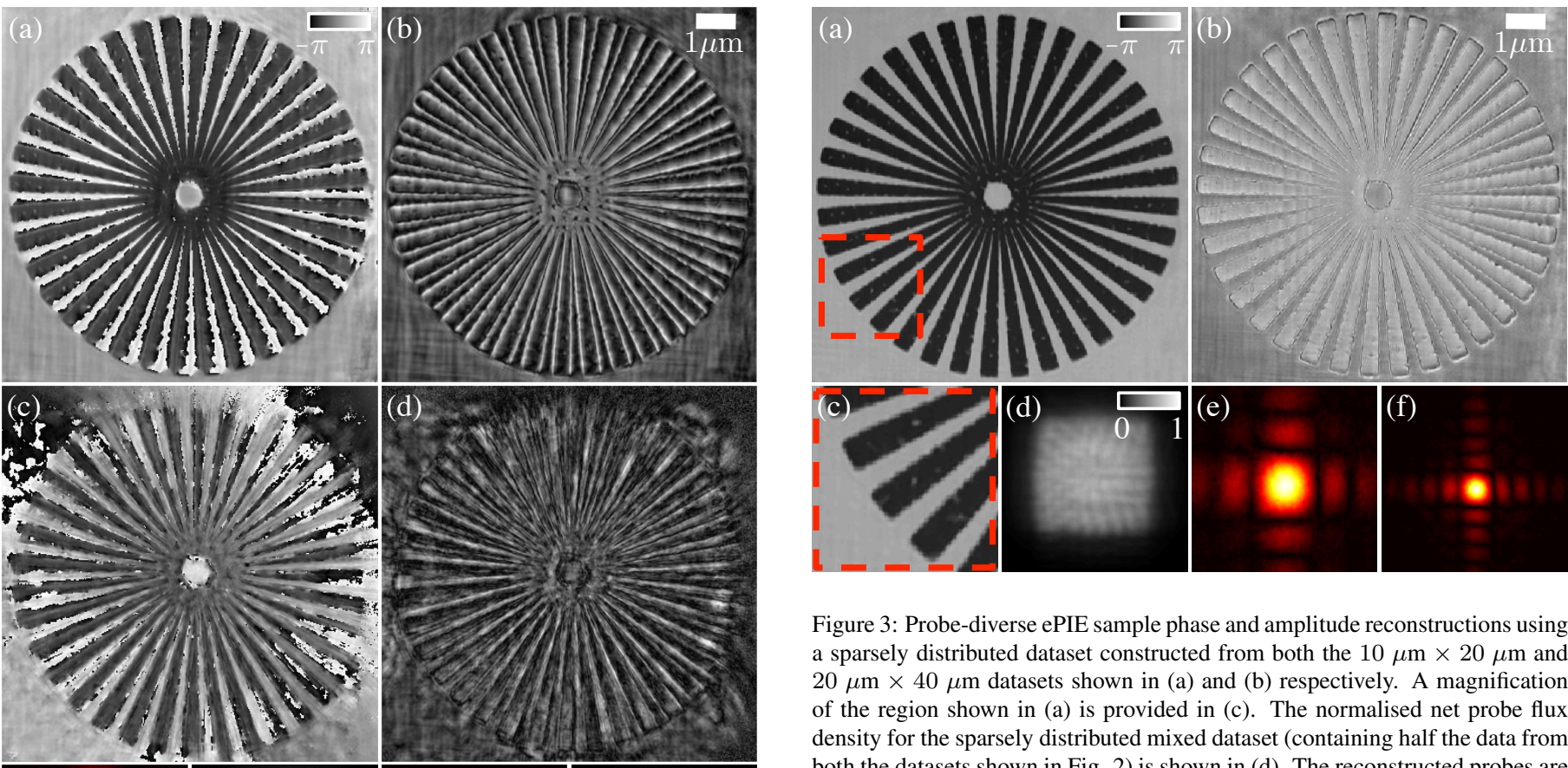

Figure 3: Probe-diverse ePIE sample phase and amplitude reconstructions using a sparsely distributed dataset constructed from both the $10 \mu \mathrm{m} \times 20 \mu \mathrm{m}$ and $20 \mu \mathrm{m} \times 40 \mu \mathrm{m}$ datasets shown in (a) and (b) respectively. A magnification of the region shown in (a) is provided in (c). The normalised net probe flux density for the sparsely distributed mixed dataset (containing half the data from both the datasets shown in Fig. 2) is shown in (d). The reconstructed probes are shown in (e) and (f).


Figure 2: Retrieved sample transmission phase and amplitude for the $10 \mu \mathrm{m} \times$ $20 \mu \mathrm{m}$ dataset using the standard ePIE algorithm are shown in (a) and (b) respectively. The reconstructed probe amplitude is shown in (e) and the normalized flux density across all probe positions is shown in (f). The corresponding ePIE sample transmission reconstructions for the $20 \mu \mathrm{m} \times 40 \mu \mathrm{m}$ dataset are shown in (c) and (d), and the probe and probe flux density shown in (g) and (h). Scale bars are common.

(c) and (d). The reconstructed probe amplitude is provided in Fig. 2 (g), with the normalized probe flux density shown in Fig. 2 (h). The erroneous sample transmission amplitude and phase variation, noted in the $10 \mu \mathrm{m} \times 20 \mu \mathrm{m}$ sample transmission reconstruction, was also present in the $20 \mu \mathrm{m} \times 40 \mu \mathrm{m}$ dataset reconstructions. The reduction in reconstruction quality compared to the $10 \mu \mathrm{m} \times 20 \mu \mathrm{m}$ dataset reconstructions may be largely attributed to the decrease in probe size and corresponding decrease in probe overlap, determined to be $53.4 \%$ between adjacent probe positions. The decrease in probe overlap resulted in a corresponding reduction in the normalized net probe flux density seen by comparing Fig. 2 (f) with (h).

The sample and both probes were then reconstructed using the probe-diverse ePIE algorithm and a mixed ptychographic dataset composed of diffraction data recorded at every odd scan position in the scanning trajectory for the $10 \mu \mathrm{m} \times 20 \mu \mathrm{m}$ and every even scan position for the $20 \mu \mathrm{m} \times 40 \mu \mathrm{m}$ datasets. This resulted in a mixed diffraction dataset of the same size as the single-probe ePIE reconstructions, with alternating probes for each subsequent sample translation. The sparsely distributed two-probe dataset resulted in a $29.8 \%$ reduction in the mean probe-overlap ratio compared to the larger probe. This reduc- tion can be seen by comparing the normalised net probe flux density in Fig. 3 (d) with the flux densities in Fig. 2.

The remaining algorithm parameters were the same as those used in the standard ePIE reconstructions. The probe-diverse sample transmission phase and amplitude are shown in Fig. 3 (a) and (b) respectively and the simultaneously reconstructed probe amplitudes are shown in (e) and (f). The erroneous amplitude and phase variation present in the single-probe reconstructions was absent in the probe-diverse reconstructions, yielding a significant qualitative and quantitative improvement in the probe-diverse sample transmission image compared to the single-probe reconstructions in Fig 2. The probe-diverse sample transmission function, used in conjunction with the projection approximation [24] yielded a sample thickness measurement of $1.6 \mu \mathrm{m}$, in close agreement with the manufactured specifications of $1.5 \mu \mathrm{m}$. It is noted that the uncertainty introduced by the erroneous phase variation in the standard ePIE phase reconstructions does not allow this measurement to be provided reliably. There is also a significant improvement in the imaging accuracy of the tungsten deposits, shown in the magnification in Fig. 3 (c). The tungsten deposits result from errors in the lithographing process and are unique to each star. The distribution of the tungsten deposits, seen in the SEM magnification in Fig. 1 (b) indicates that the reconstructed sample images are of different stars (the sample consisted of multiple copies of the star aperture). The improvements in the reconstructed sample image yielded a reduction in the $\chi^{2}$ metric for all scan positions and a $20 \%$ reduction in the mean $\chi^{2}$ value.

The sample image was then reconstructed using the probediverse ePIE algorithm and the entire dataset from both the $10 \mu \mathrm{m} \times 20 \mu \mathrm{m}$ and $20 \mu \mathrm{m} \times 40 \mu \mathrm{m}$ datasets (i.e. 626 diffraction images in total). There was no significant improvement in the sample image quality or reduction in the $\chi^{2}$ metric compared to 
the sparsely distributed data reconstructions. An additional test with the additional data provided from the $40 \mu \mathrm{m} \times 50 \mu \mathrm{m}$ probe dataset (i.e. 969 diffraction images in total) also did not yield subsequent improvement in the reconstructed sample transmission function. It appears that, for this ptychographic dataset, the limitations in the achievable sample image reconstruction quality, imposed by the recorded diffraction data, uncertainty in the sample translations $[18,14]$, and deviations from full coherence in the illuminating probe, did not yield further improvement in the reconstructed sample image with additional data or probes.

\section{Conclusion}

The use of a two-probe ptychographic system was effective in overcoming the stagnation encountered in the standard single probe scheme. In particular, when using a mixed ptychographic dataset of equal size to the standard single-probe reconstructions, the probe-diverse method was shown to yield superior results in the final sample image reconstruction compared to those obtained using the standard single-probe ePIE algorithm.

Additional ptychographic data, whether from data acquired at additional scanning trajectory points in the two-probe system, or from additional data acquired with a third distinct probe, yielded negligible improvement in the probe-diverse sample image reconstructions.

\section{Acknowledgments}

The measurements were carried out at APS beamline 34ID-C, built with US National Science Foundation grant DMR9724294 and operated by the US Department of Energy, Office of Basic Energy Sciences, under contract no. DE-AC0206CH113 I. Peterson acknowledges support from the Australian Research Council Centre of Excellence for Coherent X-ray Science. I. K. Robinson acknowledges support from the UK EPSRC under EP/I022562/1 "Phase modulation technology for X-ray imaging".

\section{References}

[1] J. Rodenburg, A. Hurst, A. Cullis, B. Dobson, F. Pfeiffer, O. Bunk, C. David, K. Jefimovs, I. Johnson, Hard-X-ray lensless imaging of extended objects, Phys. Rev. Lett. 98 (2007) 34801.

[2] A. M. Maiden, J. M. Rodenburg, An improved ptychographical phase retrieval algorithm for diffractive imaging, Ultramicroscopy 109 (2009) $1256-1262$.

[3] P. Thibault, M. Dierolf, A. Menzel, O. Bunk, C. David, F. Pfeiffer, Highresolution scanning X-ray diffraction microscopy, Science 321 (2008) 379-382.

[4] O. Bunk, M. Dierolf, S. Kynde, I. Johnson, O. Marti, F. Pfeiffer, Influence of the overlap parameter on the convergence of the ptychographical iterative engine, Ultramicroscopy 108 (2008) $481-487$.

[5] C. T. Putkunz, J. N. Clark, D. J. Vine, G. J. Williams, M. A. Pfeifer, E. Balaur, I. McNulty, K. A. Nugent, A. G. Peele, Phase-diverse coherent diffractive imaging: High sensitivity with low dose, Physical review letters 106 (2011) 013903.

[6] T. Shimobaba, T. Kakue, N. Okada, Y. Endo, R. Hirayama, D. Hiyama, T. Ito, Ptychography by changing the area of probe light and scaled ptychography, Optics Communications 331 (2014) 189-193.
[7] M. Dierolf, P. Thibault, A. Menzel, C. M. Kewish, K. Jefimovs, I. Schlichting, K. Von Koenig, O. Bunk, F. Pfeiffer, Ptychographic coherent diffractive imaging of weakly scattering specimens, New Journal of Physics 12 (2010) 035017.

[8] I. Peterson, B. Abbey, C. Putkunz, D. Vine, G. van Riessen, G. Cadenazzi, E. Balaur, R. Ryan, H. Quiney, I. McNulty, et al., Nanoscale fresnel coherent diffraction imaging tomography using ptychography, Optics express 20 (2012) 24678-24685.

[9] C. T. Putkunz, A. J. DAlfonso, A. J. Morgan, M. Weyland, C. Dwyer, L. Bourgeois, J. Etheridge, A. Roberts, R. E. Scholten, K. A. Nugent, et al., Atom-scale ptychographic electron diffractive imaging of boron nitride cones, Physical review letters 108 (2012) 073901.

[10] M. Humphry, B. Kraus, A. Hurst, A. Maiden, J. Rodenburg, Ptychographic electron microscopy using high-angle dark-field scattering for sub-nanometre resolution imaging, Nature communications 3 (2012) 730.

[11] A. D'Alfonso, A. Morgan, A. Yan, P. Wang, H. Sawada, A. Kirkland, L. Allen, Deterministic electron ptychography at atomic resolution, Physical Review B 89 (2014) 064101.

[12] Y. Shi, T. Li, Y. Wang, Q. Gao, S. Zhang, H. Li, Optical image encryption via ptychography, Optics letters 38 (2013) 1425-1427.

[13] N. Rawat, I.-C. Hwang, Y. Shi, B.-G. Lee, Optical image encryption via photon-counting imaging and compressive sensing based ptychography, Journal of Optics 17 (2015) 065704.

[14] A. Maiden, M. Humphry, M. Sarahan, B. Kraus, J. Rodenburg, An annealing algorithm to correct positioning errors in ptychography, Ultramicroscopy 120 (2012) 64-72.

[15] T. Edo, D. Batey, A. Maiden, C. Rau, U. Wagner, Z. Pešić, T. Waigh, J. Rodenburg, Sampling in x-ray ptychography, Physical Review A 87 (2013) 053850.

[16] D. Batey, T. Edo, C. Rau, U. Wagner, Z. Pešić, T. Waigh, J. Rodenburg, Reciprocal-space up-sampling from real-space oversampling in X-ray ptychography, Physical Review A 89 (2014) 043812.

[17] P. Thibault, A. Menzel, Reconstructing state mixtures from diffraction measurements, Nature 494 (2013) 68-71.

[18] F. Zhang, I. Peterson, J. Vila-Comamala, A. Diaz, F. Berenguer, R. Bean, B. Chen, A. Menzel, I. K. Robinson, J. M. Rodenburg, Translation position determination in ptychographic coherent diffraction imaging, Optics express 21 (2013) 13592-13606.

[19] M. Beckers, T. Senkbeil, T. Gorniak, K. Giewekemeyer, T. Salditt, A. Rosenhahn, Drift correction in ptychographic diffractive imaging, Ultramicroscopy 126 (2013) 44-47.

57.[20] S. Yi-Shi, W. Ya-Li, Z. San-Guo, Generalized ptychography with diverse probes, Chinese Physics Letters 30 (2013) 054203.

[21] X. Huang, M. Wojcik, N. Burdet, I. Peterson, G. R. Morrison, D. J. Vine, D. Legnini, R. Harder, Y. S. Chu, I. K. Robinson, Quantitative X-ray wavefront measurements of fresnel zone plate and $\mathrm{kb}$ mirrors using phase retrieval, Optics express 20 (2012) 24038-24048.

[22] B. L. Henke, E. M. Gullikson, J. C. Davis, X-ray interactions: photoabsorption, scattering, transmission, and reflection at $\mathrm{e}=50-30,000 \mathrm{ev}, \mathrm{z}=$ 1-92, Atomic data and nuclear data tables 54 (1993) 181-342.

[23] P. Thibault, M. Dierolf, O. Bunk, A. Menzel, F. Pfeiffer, Probe retrieval in ptychographic coherent diffractive imaging, Ultramicroscopy 109 (2009) 338-343.

[24] D. Paganin, Coherent X-ray optics, 6, Oxford University Press on Demand, 2006. 